\title{
Colchicine in the Treatment of Recurrent Oral Aphthous Ulcer -Open Clinical Trial in Bangladesh.
}

\author{
A Hassan 1, Z Ahmed 2, R Ali 3, F Ara 4, N Ahmed 5, M U Salam 6
}

\begin{abstract}
Background: Recurrent aphthous Ulceration (RAU) is characterized by necrotizing ulcers of the oral mucosa that persist, remit, and recur for variable periods of time. Despite the benign nature of the disease, RAU frequently affects quality of life as a result of long lasting and recurrent episodes of burning pain. Aim and objective: the aim of the study was to determine the efficacy of colchicine in treatment of recurrent oral aphthous ulcer. Methods: A clinical trial was conducted among 30 samples in the department of dermatology and venereology of Bangabandhu Sheikh Mujib Medical University and Dhaka Medical College Hospital, Dhaka. Study was performed among those who fulfilled the inclusion criteria. Data was collected from 1st September 2007 to 29th February.
\end{abstract}

\section{Introduction}

Recurrent Aphthous Ulceration (RAU) is an inflammatory condition of unknown etiology characterized by painful, recurrent single or multiple ulceration of the oral mucosa. 1 Aphthae like ulcers may also occur in genital mucosa. It is the most common inflammatory ulcerative.

$1 . \quad$ Ayesha

HassanAssistant professor

Department of Dermatology \& Venereology

Delta Medical College \& Hospital, Dhaka

2. Zakir Ahmed-

professor

Department of Dermatology \& Venereology

Bangabandhu Sheikh Mujib Medical University, Dhaka

3. Rubaiya Ali

professor

Department of Dermatology \& Venereology

Ad-Din Medical College \& Hospital, Dhaka

4. Ferdous Ara— Associate

professor

Department of Gynaecology \& Obstetrics

Delta Medical College \& Hospital, Dhaka 5.

Ahmed-

Nafiza

professor

Department of Dermatology \& Venereology

Dhaka Medical College \& Hospital, Dhaka

$6 . \quad$ Mahjuba Umme

Salam—_-Assistant Professor

Department of Medicne

Sylhet Women's Medical College \& Hospital, Sylhet

Corresponding author

Dr. Ayesha Hassan

simi187@yahoo.com
2008 and enrolled data was analyzed by using statistical SPSS win 13. Results: Among 30 patients, 24 (80\%) were male and $6(20 \%)$ were female. Mean age of the patients was 26.20 ( \pm 6.65$)$ years. $26(87.7 \%)$ patients found ulcer in oral mucosa and 4(13.3\%)in tongue, but previous family history was present in only $20 \%$ cases out of 30 patients. All patients experienced a marked decrease in symptoms during colchicine treatment in where; mean aphthae count was $3.27( \pm 1.05)$ before treatment and $0.43( \pm 0.68)$ during treatment. Pain score also reduced during treatment from $6.80( \pm 2.12)$ to 1.13 ( $\pm .1 .11)$ which was statistically highly significant. Conclusions: Colchicine may be a promising modality of treatment in the therapeutic armamentarium for RAU.

Condition of the oral mucosa.2 RAU has three different variants-minor aphthous ulcer, major aphthous ulcer and herpetiform ulcers.

The classic presentation of RAU is recurrent, self-limiting ulcers that mainly affect nonkeratinized oral mucosa and is characterized by necrotizing ulcers that persist, remit, and recur for variable periods of time.3,4,5,6,7 Despite the benign nature of the disease, RAU frequently affects quality of life as a result of long lasting and recurrentepisodes of burning pain.8 The etiology of recurrent aphthous ulceration is not understood.2 No principal cause has been discovered, however attacks may be precipitated by, or associated with, local trauma, stress, food hypersensitivity, hormonal changes, microorganisms, vitamin and trace element deficiencies.1 Systemic conditions including genetic predisposition, immune dysregulation, and family history might play a role in recurrent aphthous ulceration in some patients.9

A diagnosis of recurrent aphthous ulceration depends mainly on history and clinical examination. Patients with mild recurrent aphthous ulceration usually do not require any treatment for the lesion. Treatment of RAU is challenging. The two main goals of therapy are supportive symptom management and potentially, the start of systemic suppressive therapy to modify the intensity and frequency of attacks. Patients with bothersome RAU may seek medical advice and are often.
Bangladesh Medical Journal 2010 Vol. 39 No. 3

treated with topical corticosteroids to relieve symptoms. For many patients, these agents provide sufficient symptomatic therapy. However, for patients whose disease is more severe,

\section{September 2010 Issue}

Collected with a structured questionnaire by face to face interview. Informed written consent was taken from each patient before conducting interview. After diagnosis patients 
response to treatment varies, and relapse is common. Several systemic drugs have been used to treat RAU, including systemic corticosteroids, thalidomide, pentoxifylline, colchicine, and dapsone. Systemic corticosteroids offer symptomatic relief and the healing of ulcers but do not affect the disease course or provide long-term remission of RAU. Furthermore, serious risks are associated with long-termsy stemic corticosteroid use. Therefore, it is preferable to suppress RAU with corticosteroid-sparing anti-inflammatory agents. Colchicine has shown promise for the treatment of complex aphthosis.10,11,12,13 Colchicine inhibits the chemotactic activity of neutrophils and patients respond well to colchicine by decreased number and duration of lesions.11 So far no trial has been done in Bangladesh with colchicine in patients with RAU. The aim of the study was to determine the efficacy of colchicine in treatment of recurrent oral aphthous ulcer in Bangladeshi population.

\section{Materials and methods}

This study was conducted among persons who came for consultation with RAU at the outpatient departments of Dermatology and Venereology of Bangabandhu Sheikh Mujib Medical University and Dhaka Medical College Hospital, Dhaka during the period of 1st September 2007 to 29th February 2008. Inclusion criteria were: Patients with painful oral ulcers in the last 6 months, age at least 18 years or above, lesions located on reachable sites of the oral mucosa, no current treatment for ulceration or willingness to stop the current treatment. Exclusion criteria were: subjects with less than two episodes of recurrent aphthous ulcers on the previous year, history of a systemic disease that could predispose the patient to aphthous stomatitis or in which colchicine is contraindicated, under systemic or topical corticosteroid therapy or immune-modulating treatment, pregnant women or lactating mother, patients unwillingness to give informed consent to take part in the study.

Thirty patients were enrolled after matching with inclusion and exclusion criteria. Data were were given tablet Colchicine $(0.6 \mathrm{mg})$, one tablet twice daily for two months (end point) to observe the efficacy and side effects. At the time of first interview, patient was undergone oral examination and asked questions regarding any family history of RAU and socio-demographic parameters. Patients were also taught how to complete self examination at home. Each patient was asked to maintain a diary to record the presence of ulcers (yes or no) and associated level of daily pain (on a 10 point scale, where 0 indicates no pain and 10 , the worst pain ever experienced with oral -ulcers). In addition, once during each episode of ulcers, on the day, the patient experienced the worst pain he/she recorded the number of ulcers that was present and their average sizes. The size was assessed by comparing ulcer size to a chart showing 6 circles of increasing diameter between 1 and $10 \mathrm{~mm}$ and numbered 1 to 6 , respectively. Some baseline investigations like complete blood counts, serum creatinine and serum SGPT were done for exclusion of major systemic diseases in which colchicine is contraindicated. Patients were instructed to report every one month interval. After collection, data were checked for inadequacy, consistency. After cleaning data, it was analyzed by using SPSS win 13 software package. Statistical significance is set at .05 level and confidence interval at 95\% level.

\section{Results}

Among 30 patients, 24 (80\%) were male and 6(20\%) were female. Maximum number of patients were ranged from 21-30 years of age, while mean age was found $26.20( \pm 6.65)$. Mean age of 1 st onset of the aphthous ulcer was $12.4 \pm 8.73$ years. Family history of the same disease was found in $20 \%(n=6)$ patients. Of all patients $26(86.7 \%)$ had oral involvement and 4 (13.3\%) had tongue involvement. Out of all patients 21 (70.0\%) had history of trauma before development of aphthous ulcer, 18 (60.0\%) had stress full life event, 13 (43.3\%) had given history of involvement of some food, 3 (10.0\%) had given history of cessation of smoking.
Bangladesh Medical Journal 2010 Vol. 39 No. 3

Of all female patients 2 (33.3\%) had given history of relationship of their aphthous ulcer and menstrual cycle [Figure-1]. Swelling and burning were the most common presenting complaints (80\%) [Table-1]. Out of all patients 24 (80.0\%) had burning, 17 (56.7\%) had tingling, 24 (80.0\%) had swelling and 18 (60.0\%) had erythema. All patients had

\section{September 2010 Issue}

$( \pm 1.05)$ before treatment and $0.43( \pm 0.68)$ during treatment $(\mathrm{P}$ value $<0.001)$. Mean average daily pain score was 6.80 $( \pm 2.12)$ before treatment and $1.13( \pm 1.11)$ during treatment $(\mathrm{P}$ value <0.001) [Table-3]. Markedly decrease mean aphthae count was observed in the patients of different types of aphthae. Mean aphthae count decreased by $83.39 \%$, from 3.27 
complaints of pain. Six (20.0\%) had solitary lesion and 24 (80.0\%) had multiple lesions. Out of all patients mean aphthae count was 3.27
$( \pm 1.05)$ to $0.43( \pm 0.68)(\mathrm{p}<0.001)$ [Table-2]. During treatment 8 (26.7\%) patients had abdominal discomfort, 2 (6.7\%) patients had vomiting and 4 (13.3\%) had diarrhea.

Figure 1: Distribution of the patients by predisposing factors of recurrent aphthous ulcer $(n=30)$

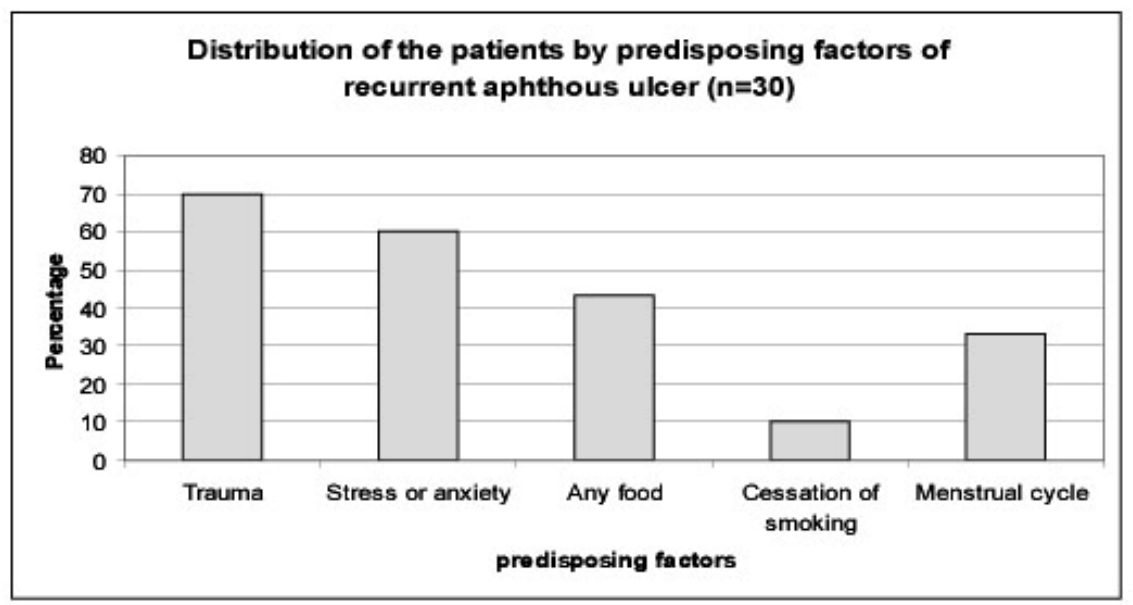

Table 1: Distribution of the patients by their presenting complaints and signs

\begin{tabular}{|c|c|c|}
\hline Clinical feature & Frequency & Percent \\
\hline \multicolumn{3}{|l|}{ Prodormal symptom } \\
\hline - Burning & 24 & 80.0 \\
\hline - $\quad$ Tingling & 17 & 56.7 \\
\hline - Swelling & 24 & 80.0 \\
\hline - Erythema & 18 & 60.0 \\
\hline Pain & 30 & 100.0 \\
\hline \multicolumn{3}{|l|}{ Lesion } \\
\hline - Soitary & 6 & 20.0 \\
\hline - Multiple & 24 & 80.0 \\
\hline \multicolumn{3}{|l|}{ Border } \\
\hline - Regular & 24 & 80.0 \\
\hline - Irregular & 6 & 20.0 \\
\hline \multicolumn{3}{|l|}{ Cluster } \\
\hline - No cluster & 24 & 80.0 \\
\hline - Small cluster & 6 & 20.0 \\
\hline Age of $1^{\text {st }}$ onset (year) & \multicolumn{2}{|c|}{$12.4 \pm 8.73(1-26) *$} \\
\hline
\end{tabular}


Table 2: Distribution of aphthae count of different types of aphthae by improvement

\begin{tabular}{|l|c|c|c|}
\hline Type of aphthae & \multicolumn{2}{|c|}{ Mean aphthae count } & Improvement (\%) \\
\hline & Before colchicine & During colchicine & \\
\hline Major & 3.43 & 0.43 & 85.71 \\
\hline Minor & 3.24 & 0.43 & 88.33 \\
\hline Herpetiform & 3.0 & 0.50 & 83.33 \\
\hline Total & 3.27 & 0.43 & 87.39 \\
\hline
\end{tabular}

Table 3: Distribution of pain score of different types of aphthae by improvement

\begin{tabular}{|l|c|c|c|}
\hline Type of aphthae & \multicolumn{2}{|c|}{ Mean pain score } & Improvement (\%) \\
\hline & Before colchicine & During colchicine & \\
\hline Major & 7.71 & 1.71 & 78.57 \\
\hline Minor & 6.33 & 0.95 & 83.21 \\
\hline Herpetiform & 8.50 & 1.00 & 90.00 \\
\hline Total & 6.80 & 1.13 & 82.58 \\
\hline
\end{tabular}

\section{Discussion}

RAU is the most common type of ulcerative disease of the oral mucosa. In this study, mean age of the patients was 26.20 ( \pm 6.65 ) years and mean age of 1 st onset of the aphthous ulcer was $12.4 \pm 8.73$ years. Lynde et al 13 showed that mean age was 41 years, and the median duration of disease before presentation was 13 years. However, they included only complex aphthosis in their study. Among 30 patients, 24 (80\%) were male and 6(20\%) were female. Fontes et al 11included 54 patients, of them 39 were female and 15 were male. Mean age of female was $44 \pm 16.8$ and male was $49 \pm 13.5$ years. Their result was not comparable with our series. This may be due to limited access to health care facilities by female patients in our country. Several hereditary and genetic predispositions involve in occurrence of RAU. It was found in one study that more than 42 percent of patients had first degree relatives with RAU.14 In the present study 6 (20.0\%) patients had family history of RAU.

Trauma, stressful life events, foods such as chocolate, coffee, peanuts, cereals, almonds, strawberries, cheese, tomatoes (even the skin of the tomatoes) and wheat flour (containing gluten), hormonal imbalance, cessation of smoking some times act as predisposing factors 7. Our study also has shown that trauma, stress or anxiety, food habits, cessation of smoking and menstrual cycle are predisposing factors. Oral mucosa was the most affected site for ulcer and was found in $88 \%$ patients in this study whereas another study found oral aphthae among $82 \%$ patients. 13

Colchicine was found to be effective in the prevention of aphthae count, daily pain reduction in major, minor and herpetiform type of RAU in most patients in our study. During treatment there was statistically significant reduction of aphthae count than earlier from $3.27( \pm 1.05)$ to $0.43( \pm 0.68)$ $(\mathrm{p}<0.001)$. Moreover, patients mean average daily pain score was also reduced from $6.80( \pm 2.12)$ to $1.13( \pm 1.11)$ during treatment. The result was comparable with the study by Katz et al10 where the mean aphthae count decreased by $71 \%$ from $3.15 \pm 0.88$ before colchicine treatment to $0.9 \pm 0.72$ during colchicine treatment $(\mathrm{p}<0.001)$. The mean pain/discomfort score decreased by $77 \%$ from 
degree was observed in all three types of aphthae in their series. After 3 months treatment with colchicine in Fontes et al 11 series, 12 patients (22\%) no longer had aphthae and was in complete remission, 22 patients (41\%) were significantly improved, since the frequency and duration of the lesions had decreased by at least $50 \%$ and the latter were no longer painful. In our series during treatment 8 (26.7\%) patients had abdominal discomfort, 2 (6.7\%) patients had vomiting and 4 (13.3\%) had diarrhoea. In Katz et al0 series four patients had mild side effects including nausea, diarrhoea, abdominal cramps, and urticaria. All side effects were transient and did not require cessation of treatment and this is similar to present study.

\section{Conclusion}

It is proposed that colchicine may be an effective option for the treatment of RAU. Ease of oral administration with minimal side effects makes this drug as a promising option to the therapeutic armamentarium for recurrent aphthous ulcer. However, this study was carried out in a small scale for a short period of time. So it may not reflect the actual scenario which can be represented. Large scale trial for a long period of time is necessary.

\section{References}

1. Natah SS, Konttinen YT, Enattah NS, Ashammakhi N, Sharkey KA, Hayrinen-Immonen R et al., Recurrent aphthous ulcers today: a review of the growing knowledge. Jnt. J. Oral Maxillofac. Surg. 2004; 33:221-234.

2. Rogers RS III. Recurrent aphthous stomatitis: clinical characteristics and associated systemic disorders. Semin Cutan Med Surg. 1997;16(4):278-283.

3. Vincent SD, Lilly GE. Clinical, historic and therapeutic features of aphthous stomatitis. Oral Surg Oral Med Oral Pathol. 1992;74:79-86.

4. Woo SB, Sonis ST. Recurrent aphthous ulcers: a review of diagnosis and treatment. J Am Dent Assoc. 1996;127:1202-13.

5. Ship JA, Chavez EM, Doerr PA, Henson BS. Recurrent Aphthous stomatitis. Quintessence Int. 2000;31:95-112.

6. Casiglia JM. Recurrent aphthous stomatitis: etiology, diagnosis, and treatment. Gen Dent 2002;50:157-66. management of recurrent aphthous stomatitis: a consensus approach. J Am Dent Assoc. 2003;134:200-7.

8. Chiappelli F, Cajulis,OS. Psychobiologic views on stressrelated oral ulcers. Quintessence Int. 2004;35:223-7.

9. Ruah CB, Stram JR, Chasin WD. Treatment of severe recurrent aphthous stomatitis with colchicine. Arch Otolaryngo l Head Neck Surg.1988;114(6):671-675.

10. Katz J, Langevitz P, Shemer J, Barak S, Livneh A. Prevention of recurrent aphthous stomatitis with colchicine: an open trial. J Am Acad Dermatol. 1994; 31(3, pt 1):459-461.

11. Fontes V, Machet L, Huttenberger B, Lorette G, Vaillant L. Recurrent aphthous stomatitis: treatment with colchicine: an open trial of 54 cases [article in French]. Ann Dermatol Venereol. 2002;129(12):1365-1369.

12. Altinor S, Ozturkcan S, Hah MM. The effects of colchicine on neutrophil function in subjects with recurrent aphthous stomatitis. J Eur Acad Dermatol Venereol. 2003;17(4):469470.

13. Carrie B. Lynde, ; Alison J. Bruce, ; Roy S. Rogers III,. Successful Treatment of Complex Aphthosis With Colchicine and Dapsone. Arch Dermatol , 2009; 145:273-276.

14. Zabarski R, Kalderon S, Klein T, Weinberger A. Close association of HLA-B51 in persons with recurrent aphthous stomatitis. Oral Surg Oral Med Oral Pathol 1992;74:455 